\title{
Mutations in mammalian tolloid-like 1 gene detected in adult patients with ASD
}

\author{
Paweł Stańczak ${ }^{1}$, Joanna Witecka ${ }^{2}$, Anna Szydło ${ }^{2}$, Ewa Gutmajster ${ }^{2}$, Małgorzata Lisik ${ }^{2}$, \\ Aleksandra Auguściak-Duma ${ }^{2}$, Maciej Tarnowski ${ }^{2}$, Tomasz Czekaj ${ }^{2}$, Hanna Czekaj ${ }^{2}$ and \\ Aleksander L Sieroń*,2 \\ ${ }^{1}$ Swietokrzyskie Center for Cardiology, Regional Hospital, Kielce, Poland; ${ }^{2}$ Department of General and Molecular \\ Biology and Genetics, CoE for Research and Teaching of Molecular Biology of Matrix and Nanotechnology, \\ CoE Network, BioMedTech 'Silesia', Medical University of Silesia, Katowice, Poland
}

Atrial septal defect (ASD) is an incomplete septation of atria in human heart causing circulatory problems. Its frequency is estimated at one per 10000 . Actions of numerous genes have been linked to heart development. However, no single gene defect causing ASD has yet been identified. Incomplete heart septation similar to ASD was reported in transgenic mice with both inactive alleles of gene encoding mammalian zinc metalloprotease a mammalian tolloid-like 1 (tII1). Here, we have screened 19 ASD patients and 15 healthy age-matched individuals for mutations in TLL1 gene. All 22 exons were analyzed exon by exon for heteroduplex formation. Subsequently, DNA fragments forming heteroduplexes were sequenced. In four nonrelated patients, three missense mutations in coding sequence, and one single base change in the $5^{\prime}$ UTR have been detected. Two mutations (Met182Leu, and Ala238Val) were detected in ASD patients with the same clinical phenotype. As the second mutation locates immediately upstream of the catalytic zinc-binding signature, it might change the enzyme substrate specificity. The third change, Leu627Val in the CUB3 domain, has been found in an ASD patient with interatrial septum aneurysm in addition to ASD. The CUB3 domain is important for substrate-specific recognition. In the remaining 15 patients as well as in 15 reference samples numerous base substitutions, deletions, and insertions have been detected, but no mutations changing the coding sequence have been found. Lack of mutations in relation to ASD of these patients could possibly be because of genetic heterogeneity of the syndrome. European Journal of Human Genetics (2009) 17, 344-351; doi:10.1038/ejhg.2008.175; published online 1 October 2008

Keywords: atrial septal defect; DNA sequencing; gene mutations; heart defects; heteroduplex analysis; mammalian tolloid-like 1

\section{Introduction}

Congenital heart defects in general are related to aberrant development of the circulation system in a fetus. The

*Correspondence: Dr AL Sieron, Department of General and Molecular Biology and Genetics, Medical University of Silesia in Katowice, ul. Medyków 18, Bldg. C-1, 40-752 Katowice, Poland.

Tel: + 4832208 8394; Fax: + 4832208 8382;

E-mail: alsieron@sum.edu.pl

Received 5 May 2008; revised 16 July 2008; accepted 29 August 2008; published online 1 October 2008 defects might result either from factors damaging the fetus during development or they can be of genetic background. Some environmental factors, drugs, alcohol abuse, and infections contracted by the mother before, or during pregnancy (eg, rubella) may alter heart development. ${ }^{1-6}$ However, environmental and maternal factors have been linked to approximately $2 \%$ of congenital heart defects. ${ }^{7}$ In a significant number of heart abnormalities coexistence of a few anomalies has been shown in different parts of the circulation system indicating genetic factors. ${ }^{1,8}$ 
Chromosomal anomalies and single gene defects, however, could be attributed to the background of $8 \%$ of congenital heart defects. ${ }^{7,9-11}$ Thus, the background of most of the congenital heart defects is still poorly understood.

The heart itself and septation of the large vessels begin at the fourth week of fetal development, but the process is not completed until shortly after birth. Septation anomalies are linked to abnormal development of endocardial cushions. They are necessary for completeness of interatrial as well as interventrical septa. Incomplete interatrial septum is a common feature of such genetic disorders as Noonan, Turner, and Holt-Oram syndromes. ${ }^{10-12}$

The condition studied here is called atrial septal defect (ASD) and has some features common with the other just listed heart-related syndromes. Currently, the defects related to ASD are corrected during puberty. However, in the past, when it was untreated, the patients often suffered from numerous severe cardiovascular and pulmonary complications.

In recent years, results of research with the use of transgenic mice models revealed that a gene named tolloid-like 1 (tll1) is critical for septa development in the mouse heart. ${ }^{13}$ The mice lacking active TLL1 died at midgestation because of severe blood circulation failure. The tll1 gene product is a metalloprotease with domain structure identical to one of the alternatively spliced variants of the bmp-1 gene product, mammalian tolloid (mTLD). The mTLD was detected in mammals and some other invertebrates. ${ }^{14-18}$ The homologous protein BMP-1 was originally reported by Wozney et $a l^{19}$ in a context of ectopic bone formation in rats when protein extracts from demineralized bovine bones were used. ${ }^{20}$ Subsequently, based on protein domain structure it was classified in the family of astacin, a group of metalloproteases involved in embryo development of different species. ${ }^{17,21-23}$ It is also commonly accepted that BMP-1 is important for numerous physiological activities acting in seemingly unrelated processes, such as extracellular matrix quality control involving formation and activation of numerous factors that act in the regulation of different genetic programs. ${ }^{17-21,23-32}$

An investigation of different mouse model with ventrical spetum defect (VSD) but with active tll1 gene indicated that a different mechanism must be involved, possibly related to abnormal cell proliferation and differentiation. ${ }^{33,34}$ Developmental defects of $t l l 1^{-1-}$ embryos seemed to be exclusively restricted to the heart. ${ }^{13}$ The comparison of the defects with expression of the tll1 revealed, in normal mice embryo, its elevated expression levels during heart septation. Thus, it is possible that the expression of TLL1 in humans could also be critical for correct heart positioning and its proper septation.

Here, we have tested 19 adult individuals with clinically confirmed ASD by karyotyping and screening of the TLL1 gene for mutations by heteroduplex detection followed by DNA sequencing.

\section{Materials and methods Patients}

Hospital records of more than 100 cardiological patients have been reviewed for ASD cases. Twenty individuals have been identified who fulfill the requirements of this project. All of them were informed about procedures related to the study. The procedures were conducted in accordance with the Institutional Guidelines and Regulations and with the Guidelines for the Conduct of Research Involving Human Subjects approved by the appropriate institutional review board (IRB) Bioethical Committee of the Silesian Medical University in Katowice, Poland, No. L.dz.NN-013-339/03. In addition, appropriate informed consent was obtained from the human volunteers.

Finally, 19 individuals agreed to participate in the study and they all have been surveyed for medical and family health history. On the basis of the survey's results and medical records they were divided into three groups (Table 1).

As a reference, blood samples from 15 age-matching volunteers (11 females and 4 males) were used to isolate DNA for genetic analyses. None of the volunteers have had inherited developmental heart conditions.

\section{Samples collection and handling}

From each patient two blood samples were drawn. One $3 \mathrm{ml}$ sample was mixed with heparin and used for cytogenetic diagnosis to exclude possible chromosomal anomalies. The methods for lymphocytes isolation and preparation for karyotyping were the classical methods described before. ${ }^{35,36}$ Slides were examined with a Zeiss Axioskop microscope. Images were obtained using a Sony ExwaveHAD digital camera and MultiScanBase software (Computer Scanning System Ltd., PL) with the karyotype option, enabling manual karyotyping. For each patient, a total of 15 metaphases in each of five prepared karyograms were examined to determine the number of chromosomes.

The second sample of blood $(5.0 \mathrm{ml})$ was mixed with EDTA and used for the isolation of genomic DNA.

\section{PCR conditions}

The DNA for mutation detection was isolated with the use of commercial blood mini-kit (A\&A Biotechnology, Gdansk, Poland) according to supplier instructions. The quality of purified genomic DNA was verified by spectrometry at $A_{260 / 280}$ and agarose gel electrophoresis.

Fragments of the TLL1 gene were amplified using pairs of primers designed to complement intronic sequences flanking coding sequences (Table 2). The conditions for amplification PCR were as follows: step (1) denaturation at $95^{\circ} \mathrm{C}$ for $4 \mathrm{~min}$, step (2) denaturation at $95^{\circ} \mathrm{C}$ for $30 \mathrm{~s}$, 
Table 1 Classification of ASD patients based on their individual clinical symptoms

\begin{tabular}{|c|c|c|c|}
\hline Patient number & $\operatorname{Sex}(F / M)$ & Patient's age (years) & Additional defects \\
\hline 3 & M & 51 & ND \\
\hline 5 & $\mathrm{M}$ & 55 & ND \\
\hline $10^{\mathrm{a}}$ & M & 35 & ND \\
\hline 11 & M & 52 & ND \\
\hline 15 & $\mathrm{~F}$ & 57 & ND \\
\hline \multicolumn{4}{|c|}{ Group 2: ASD with diagnosed aneurysm of interatrial septum and other defects } \\
\hline 1 & $\mathrm{M}$ & 70 & $\begin{array}{l}\text { Aneurysm, heart attack, cerebral stroke, atrial fibrillation, and cardiac pacemaker } \\
\text { implantation }\end{array}$ \\
\hline 4 & M & 55 & Heart attack \\
\hline 6 & M & 45 & Hypertrophic cardiomyopathy \\
\hline 9 & M & 72 & Aneurysm of interatrial septum sinus bradycardia \\
\hline 12 & $\mathrm{~F}$ & 58 & Asymptomatic aneurysm of interatrial septum \\
\hline 2 & $\mathrm{M}$ & 52 & Atrial fibrillation, cardiac pacemaker implantation \\
\hline 8 & M & 51 & Atrial fibrillation, cardiac pacemaker implantation \\
\hline 14 & $\mathrm{~F}$ & 56 & Cardiac pacemaker implantation \\
\hline 16 & $\mathrm{~F}$ & 67 & Atrial fibrillation \\
\hline
\end{tabular}

ASD, atrial septal defect; $F$, female; $M$, male; ND, Not determined.

${ }^{a}$ This patient was only the one who was classified with ASD type I. All the other patients had ASD type II.

step (3) annealing at the appropriate primer pair melting temperature $\left(\mathrm{T}_{\mathrm{m}}\right)$ (for details see Table 2) for $30 \mathrm{~s}$, step (4) elongation at $72^{\circ} \mathrm{C}$ for $1 \mathrm{~min}$ per each $1250 \mathrm{bp}$ of the amplified DNA fragment. The procedure was repeated 34 times from steps (2) to (4). Following the last annealing, the elongation time at $72^{\circ} \mathrm{C}$ was extended up to $7 \mathrm{~min}$. The PCR products were purified using method provided in the sequencing manual by Applied Biosystems. The DNA concentration was determined spectrophotometrically at $A_{260}$. The reference DNA was isolated from the blood of healthy individuals. The DNA reference fragments were obtained with the corresponding primers under the conditions used for patients' samples and subjected to heteroduplex analysis and DNA sequencing, when necessary except for the exons with mutations detected in patients that were sequenced entirely.

\section{Heteroduplex formation and analysis}

Equal amounts (200 ng) of each, the reference and corresponding patients' DNA fragments, in $4 \mu \mathrm{l}$ were mixed in the final volume of $10 \mu \mathrm{l}$ supplemented with one-tenth volume of $0.5 \mathrm{M} \mathrm{KCl}$, and nuclease-free water when needed. The samples were handled according to the protocols recommended by the Transgenomic Inc., as follows: melting of the DNA at $95^{\circ} \mathrm{C}$ for $2 \mathrm{~min}$ and then cooling the mixture from 95 to $85^{\circ} \mathrm{C}$ at the rate $2.0^{\circ} \mathrm{C}$ per second and from 85 to $25^{\circ} \mathrm{C}$ at the rate $0.5^{\circ} \mathrm{C}$ per second.
Subsequently, the mixture was subjected to the enzymic assay using Surveyor kit (Transgenomic Inc.). Briefly, the $400 \mathrm{ng}$ of hybridized DNA in the volume $4-15 \mu \mathrm{l}$ was mixed with $0.5 \mu \mathrm{l}$ of Surveyor Nuclease $\mathrm{S}$ and $0.5 \mu \mathrm{l}$ Surveyor Enhancer, and water, when needed, and incubated at $42^{\circ} \mathrm{C}$ for $40 \mathrm{~min}$. The cleavage was terminated by mixing the sample with one-tenth volume of the Stop Solution supplied by the manufacturer in the assay kit. To analyze the Surveyor Nuclease S digestion products by agarose gel electrophoresis one-sixth volume of a $6 \mathrm{X}$ loading dye buffer (10 mM Tris- $\mathrm{HCl}(\mathrm{pH} 8.0), 10 \mathrm{~mm}$ EDTA $(\mathrm{pH} 8.0), 50 \%(\mathrm{w} / \mathrm{v})$ sucrose, $0.15 \%(\mathrm{w} / \mathrm{v})$ bromophenol blue) was added to each sample. For each digestion/loading dye mixture, the entire volume of sample was loaded onto an agarose gel of an appropriate percentage (1.5-1.7\%) and separated electrophoretically. The mixture of $\lambda$ DNA cleaved with HindIII and $\phi \mathrm{X}$ cleaved with HaeIII (Finnzymes) was used as the DNA length marker. The gels were developed at $5 \mathrm{~V} / \mathrm{cm}$ until the bromophenol blue had reached two-thirds of the length of the gel. To visualize the bands, an illumination of the gel using a UV transilluminator $(250-300 \mathrm{~nm})$ was performed and the pictures were taken.

\section{DNA sequencing}

Samples revealing heteroduplex formation with the corresponding fragment of reference DNA were subjected to 
Table 2 PCR primers and conditions used for amplification and sequencing of the TLL1 gene

\begin{tabular}{|c|c|c|c|c|}
\hline Exon number & $\begin{array}{l}\text { Primers positions } \\
\quad \text { from-to }\end{array}$ & $\begin{array}{l}\text { Product length } \\
\quad(b p)\end{array}$ & Primer sequence (length in $b p$ ) & $\begin{array}{l}\text { Primer annealing } \\
\text { temperature }\left({ }^{\circ} \mathrm{C}\right)\end{array}$ \\
\hline 1 & $305-1626$ & 1322 & $\begin{array}{l}\text { CACgTACATCATCTATTAATTgCTgTTTgC (30) } \\
\text { TCCCACTggCACAgAACTCTAgTCAACTg (29) }\end{array}$ & 58 \\
\hline Int. 1 & $644-1626$ & 983 & $\begin{array}{l}\text { TgCAggCTTTAAggTCTCgCggCgTAg (28) } \\
\text { TCCCACTggCACAgAACTCTAgTCAACTg (29) }\end{array}$ & 62 \\
\hline 2 & $116547-118357$ & 1811 & $\begin{array}{l}\text { ggTAgCTAATATTTgTCCTgTAAC (24) } \\
\text { CTCTACTgTCCAgAATgCgTTgTC (24) }\end{array}$ & 60 \\
\hline 3 & $119808-120855$ & 1048 & $\begin{array}{l}\text { gCAgAACAACTTgCTgTCATTCTATgTAg (29) } \\
\text { ATgTACTTCAAgCATTTAATTAgTAATCCATC }(32)\end{array}$ & 52 \\
\hline 4 and 5 & $121553-122696$ & 1144 & $\begin{array}{l}\text { gCTTTTgTTCAAgTATgTAATATAACTgAA (29) } \\
\text { ACTgTgATCAAAgAATACACATACCTAgTAT (31) }\end{array}$ & 51 \\
\hline 6 & $130574-131341$ & 768 & $\begin{array}{l}\text { ggATACCAgAAACTAgTCATATgTAgTAAT (30) } \\
\text { CCTggTATAACACAgATTCCTATggTA (27) }\end{array}$ & 52 \\
\hline 7 & $135107-135828$ & 722 & $\begin{array}{l}\text { CAATATgATggTAgAgTgAAgACAgATgC (29) } \\
\text { ACACAgTATAAAgCACAAgTCgCCTAgATT (30) }\end{array}$ & $65\left(-0.5^{\circ} \mathrm{C}\right.$ per cycle $)$ \\
\hline 8 & $141561-142212$ & 652 & $\begin{array}{l}\text { GAATgTCATCATTgAgTTTATACTAg (26) } \\
\text { ATCATgCAACTATTTAATTAATgACTATCA (30) }\end{array}$ & 50 \\
\hline 9 & $152432-153304$ & 873 & $\begin{array}{l}\text { ATTTCTCTTATAATCTTATTCggTgATTCAC (31) } \\
\text { TCTAACСТCTTCATATggTCgTCTAg (26) }\end{array}$ & 51 \\
\hline 10 & $166465-167014$ & 550 & $\begin{array}{l}\text { ACTTCTCCTgACATTgTAAAACAgTTA (27) } \\
\text { CСTAgTATgTACCTCATAAgTTAAgCTAg (29) }\end{array}$ & 50 \\
\hline 11 & $169104-169695$ & 592 & $\begin{array}{l}\text { CATCTTACTTCTACATATggTAACAATA (28) } \\
\text { gATATTATTACATCACTgATACTAgACAA (29) }\end{array}$ & 50 \\
\hline 12 & $170467-171296$ & 830 & $\begin{array}{l}\text { CTACATTACAACCAgTCAACTTTgAT (26) } \\
\text { CTgACCCgTCATCTAATAACACAAC (25) }\end{array}$ & 58 \\
\hline 13 & $182254-183708$ & 1455 & $\begin{array}{l}\text { ggATATTACAgTgTACgTTCATgAgAAACA (30) } \\
\text { gACAggATACTCCATAATCTCACTCTTCA (29) }\end{array}$ & 51 \\
\hline 14 & $184178-184802$ & 625 & $\begin{array}{l}\text { gCAgCAACATgAATTTgTATACgTTATCCA (30) } \\
\text { ATCAgCTgTgACTgTgAATTgAgTCTTACT (30) }\end{array}$ & 52 \\
\hline 15 & $187056-187662$ & 607 & $\begin{array}{l}\text { TCTAAAgATAAgAAAACTgAgACCTAgA (28) } \\
\text { TgAgCAgTAAGACAAAgAAAgAATACAATg (30) }\end{array}$ & 50 \\
\hline 16 & $192768-194033$ & 1266 & $\begin{array}{l}\text { TggACAAgCTATATAAAAATCTCTgAACT (29) } \\
\text { ACAAACCCTAgACATTgTAAATCTCAT (27) }\end{array}$ & 58 \\
\hline 17 & $201974-202547$ & 574 & $\begin{array}{l}\text { gAggATTACAAgTTATTATCgTAgCg (26) } \\
\text { CTAATgCCATCTATgTgCgAAgTAC (25) }\end{array}$ & 53 \\
\hline 18 & $204748-205588$ & 841 & $\begin{array}{l}\text { ATTCAAgTgTATATTTAACTAAgAgAgA (28) } \\
\text { CAggATAAgAAACCCTAgAggAgAACAACg (30) }\end{array}$ & 50 \\
\hline 19 & $218070-218860$ & 791 & $\begin{array}{l}\text { AggATAATgTTCAACgggTTCTATgAT (27) } \\
\text { CAAAgATCTTAAggTAggATgCTgTATggT (30) }\end{array}$ & 50 \\
\hline 20 & $226477-227111$ & 635 & $\begin{array}{l}\text { CCAgTTgTggAAAACATATCTgACg (25) } \\
\text { gAAATTATACCTACTgATTTCATCggT (27) }\end{array}$ & 58 \\
\hline $21 / 22$ & $227995-231411$ & 3417 & $\begin{array}{l}\text { gACAAgTATTCCCAAACATgCgTTAC (26) } \\
\text { ACTgTgACATATTAAAATgAgCgACA (26) }\end{array}$ & 57 \\
\hline Int. 21 & $227995-229978$ & 1984 & $\begin{array}{l}\text { gACAAgTATTCCCAAACATgCgTTAC (26) } \\
\text { gTTgTATgCTCATggACAggTAACAgAC (28) }\end{array}$ & 55 \\
\hline
\end{tabular}

Int., primer for starting sequencing PCR inside of exons 1 or 21 .

DNA sequencing. ${ }^{37}$ Sequencing PCR was conducted using reagents supplied by Applied Biosystems under conditions recommended by the manufacturer. Briefly, the PCR products were purified by ethanol precipitation. The precipitated DNA was solubilized in the Hi-Di Formamide (Applied Biosystems) denatured at $95^{\circ} \mathrm{C}$ for $5 \mathrm{~min}$, cooled and analyzed using ABI PRISM 310 Genetic Analyzer (Applied Biosystems). The sequence obtained was verified using the ChromasPro software. Sequence alignment was obtained with the use of MegAlign software in DNA-Star package v. 5.0, Lasergene. The reference sequence for TLL1 used here was AC097502.3.1.168254 (GenBank). 


\section{Results}

As no chromosomal anomalies were detected by G-banding karyotype analysis in any of the ASD patients, further analyses focused on heteroduplex detection and subsequent DNA sequencing.

\section{Heteroduplex formation frequency}

All amplified PCR fragments were subjected to the DNA heteroduplex formation analysis. In two fragments containing exons 3 and 11, no heteroduplex formation was detected in any of the ASD individuals as well as in all 15 reference samples.

In all patients, mismatches were detected in gene fragments containing exons $1,5,12,14,19$, and 20 . The DNA fragments containing exons $2,4,6-10,13,15-18,21$, and 22 revealed various changes regarding the number of formed heteroduplexes as well as the number of mismatches in a single fragment. However, in 122 (34\%) heteroduplexes found in patients, as well as in all heteroduplexes detected in reference samples, their sequencing excluded mismatches, indicating that the heteroduplex detection method also generated false-positive results. The remaining $66 \%$ of the heteroduplexes detected in patients resulted from changes located mostly in the intronic sequences. Some changes were located within the distance of $50 \mathrm{bp}$ from the putative RNA splicing sites. Finally, 30 changes in the introns were confirmed by sequencing. They were substitutions: A to $G$ (13), G to A (14), T to G (1), A to T (1), and C to T (1). The remaining changes detected in the heteroduplexes could not be verified by sequencing, as they were probably located in regions out of readable sequences.

\section{Potential polymorphic changes in ASD patients}

In the DNA gene fragment containing the exon 19 in six patients from group 1, in three patients from group 2, and in two patients from group 3 as well as in control sample, a polymorphic change A to $G$ at the position 218697 was detected. Another polymorphic change of $\mathrm{T}$ to $\mathrm{C}$ at position 218734 of the same fragment was found in four patients from group 1 , in three individuals from group 2 , in three patients from group 3 , and in the control sample. In addition, a polymorphic change in two patients, one from group 1 and the other one from group 2 as well as in the reference sample was identified at the position 218441 ( $\mathrm{T}$ to A). There was also a single base deletion ( $\mathrm{T}$ del. 218443 ) in four patients from group 1, in two individuals from group 2, and in two patients from group 3. Double-base insertion (TA ins. 218328-9) has been detected in four patients from group 1, in two patients from group 2, and in two patients from group 3 .

\section{Mutations changing sense of codons}

In all examined exons 4 changes detected by heteroduplex analysis were confirmed by DNA sequencing (Figure 1).

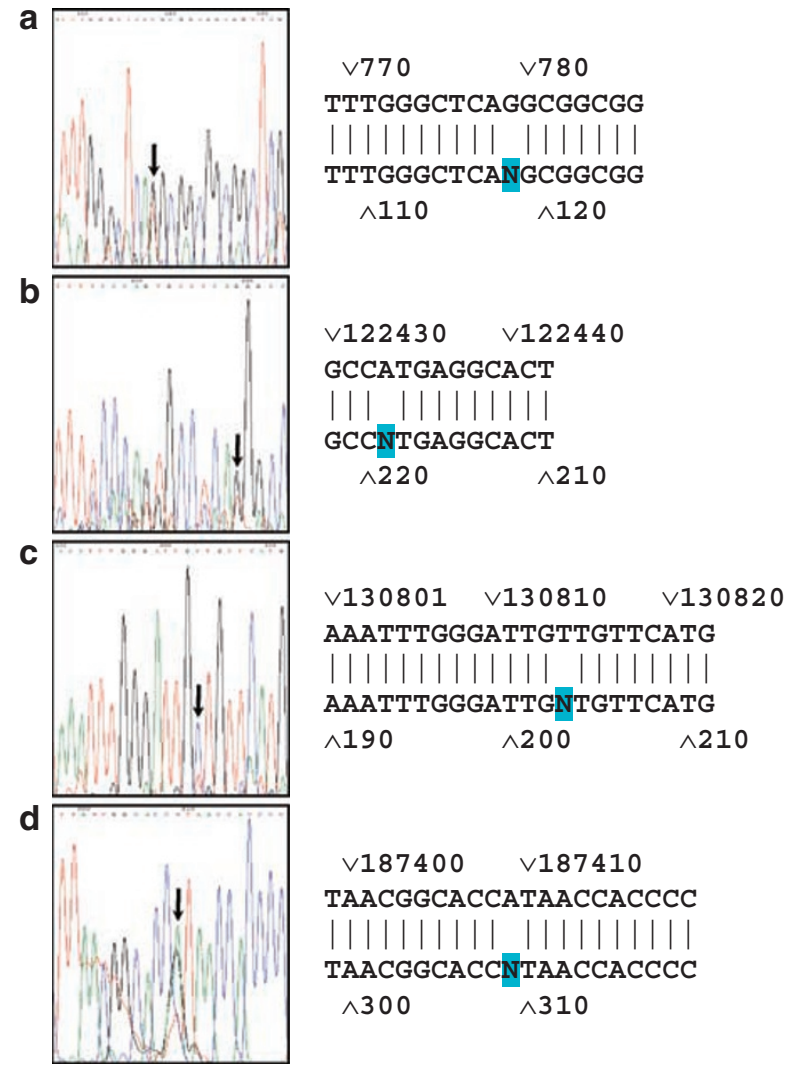

Figure 1 Heterozygous mutations detected in ASD patients. (a) A change G:G/T at position 779 detected in patient no. 17 from group 2 with ASD, aneurysm of interatrial septum and other defects. (b) A change $A: A / C$ at position 122433 (1191 in CDNA) detected in patient no. 10 from group 1 with ASD symptoms alone. In the presented case, the sequence was obtained with the use of reverse primer to sequence the fragment starting at the position 121553 (exons 4 and 5). (c) A change $T: C$ at position 130814 (1360 in CDNA) detected in the patient no. 1 from group 2 with ASD, aneurysm of intersinal septum and other defects. (d) A change A:A/G at position 187409 (1885 in CDNA) detected in the patient no. 9 from group 2 with ASD, aneurysm of intersinal septum and other defects. Arrow in each panel indicates the detected changes.

The DNA fragment containing the exon 1 (305-1625) from one patient from group 2 revealed one mismatch $(\mathrm{G} / \mathrm{T})$ in the $5^{\prime} \mathrm{UTR}$ at position 779 (Figure 1a). The change, however, is not located within the Kozak consensus sequence and it was earlier annotated as SNP (GenBank AC097502.3.1.168254).

In the fragment containing exon 5, a heterozygous base substitution A:C at position 122433 (Figure 1b) (1191 in the cDNA; NM_012464) changing a codon for Met to codon for Leu at position 182 was detected. Another mutation was detected in the fragment containing exon 6 in one patient from group 2. It was a change from $\mathrm{T}$ to $\mathrm{C}$ at position 130814 changing valine 238 to alanine (Figure 1c). The last change detected during study reported here was a mutation in the gene fragment covering exon 
a Astacin

H. Tolloid/BMP-1

H. Tolloid-like 1

H. Tolloid-like 2

H. Tolloid-like 1M

H. Meprin- $\alpha$

H. Meprin- $\beta$

b Astacin

H. Tolloid/BMP-1

H. Tolloid-like 2

H. Tolloid-like 1

H. Tolloid-like 1M

SpAN

H. Meprin- $\alpha$

H. Meprin- $\beta$

H. $M M P-9$

H. $M M P-13$

H. MMP -3

H. $M M P-2$
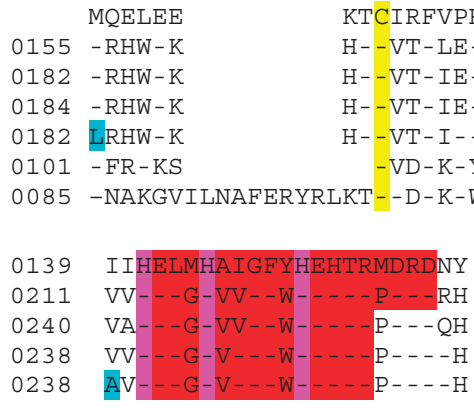

0188 -V--IG-- - - H--QS-P-- - E-

$\begin{array}{ll}0153 & -\mathrm{E}-\mathrm{IL}-\mathrm{L}----\mathrm{QS}-\mathrm{T}---\mathrm{D}- \\ 0150 & \mathrm{VQ}-\mathrm{FL}--\mathrm{L}--\mathrm{W}--\mathrm{QS}-\mathrm{S}---\mathrm{D}-\end{array}$

0399 AA--FG--L-LD-SSVPEALMYP

0220 AA--FG-SL-LD-SKDPGALMFP

0215 AA--IG-SL-LF-SANTEALMYP

0401 AA--FG--M-LE-SQDPGALMAP

C H. Tolloid/BMP-1 CUB2 444 GHIOSPNYPDDYRPSK

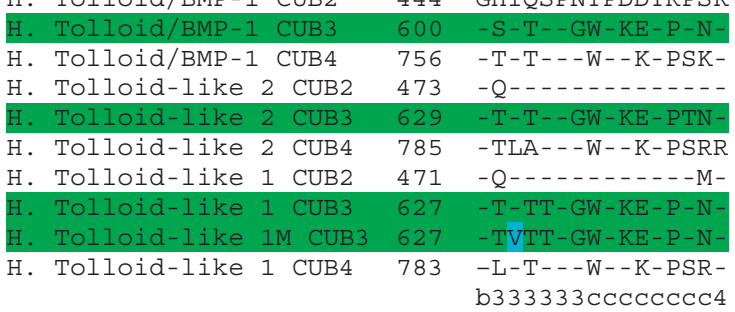

3 and $4=\beta$-strands 3 and 4

$\mathrm{b}$ and $\mathrm{c}=$ loops $\mathrm{b}$ and $\mathrm{c}$

Figure 2 Comparison of conserved amino acid sequence domains mutated in TLL1 with the corresponding domains in selected metzincins. (a) N-terminal end of the catalytic domain, (b) active centers of selected metalloproteases from astacin family and other selected metzincins. (c) N-terminal end of the CUB3 domain. Symbols: $\mathrm{H}$, amino acid sequence of human polypeptides; M, mutated TLL1; dashes, identical residues as in the polypeptide aligned at the top of the alignment. Colors: yellow, conserved cysteines; pink, histidines coordinating zinc in the active center of the enzyme; red, amino acid sequence in extended catalytic center; green, CUB3 domains in different enzymes; blue, mutations detected in ASD patients.

15 in one patient from group 2 at position 187409 (2532 in cDNA; NM_012464) changing A to G and resulting in a codon 629 for isoleucine being substituted to codon for valine (Figure 1d).

\section{Discussion}

Recently, numerous reports described mutations at the level of single genes, including NKX 2.5 and GATA4 in relation to heart developmental anomalies. ${ }^{38}$ Therefore, the lack of structural and numerical chromosomal abnormalities observed here is not surprising. Lack of mutations in exons 3 and 11 indicates that the structural parts of the protein encoded by these exons must be vital for the development. Indeed, the exon 3 encodes amino acids building central portion of the latency propeptide. Thus, we postulate that any change in this part of the peptide could alter the structure by the mean of its miss folding, which in turn could lead to serious problems in specific activation of the enzyme by putative furins. ${ }^{14}$
Retention of the latency peptide would be a reason for the lack of activity of TLL1.

The exon 11 encodes amino acids $421-460$ being a part of the CUB1 domain. For proteinases homologous to TLL1, such as mTld and BMP-1, it has been shown that the CUB1 domain is critical for their secretion from the cells. ${ }^{39,40}$ Lack of this domain or shuffling it into a different part of the recombinant protein led to the retention of such an aberrant product in the cytoplasm. ${ }^{39}$ Therefore, it is possible that mutations in the exon 11 would cause a complete lack or at least poor secretion of TLL1 in a human and to a natural abortion of the embryo because of the developmental abnormalities as has been reported for transgenic mice null for $t l l 1 .^{13}$

The mutation in codon at position 182 may affect conversion of the zymogen to the active enzyme. The methionine 182 is the first residue in the catalytic domain, which is conserved among all the astacins including maprins $\alpha$ and $\beta$ (Figure 2a). It is also the first amino acid in the sequence with positions conserved for two cysteines 
in all known members of the astacin family (Figure 2a). Therefore, mutation of the hydrophobic residue such as Met182 to an aliphatic Leu residue, which occupies place in the first $\alpha$ helix of the catalytic domain ${ }^{41}$ might have a consequence in altering the structure of catalytic domain and affect the activity of TLL1 on its native substrate, chordin.

Another mutation caused a change of residue at position 238, which is located within the catalytic domain, just before the zinc-binding signature (Figure $2 \mathrm{~b}$ ). The structure of this region is critical for substrate accommodation of the catalytic cleft. This cleft in all tolloids is deeper than the cleft in astacin. ${ }^{42,43}$ A deeper cleft limits substrates that may fit into the active center of the enzyme to hydrolyze the specific peptide bound. ${ }^{42,43}$ The conserved two residues preceding the zinc-binding signature in proteases classified in astacin family are always isoleucine or valine at first position and isoleucine, alanine, valine, glutamic acid or aspartic acid at second position. In other metzincins, such as MMPs, the two corresponding residues located immediately upstream of the zinc-binding signature are alanines. Substitution of the two Ile residues in astacin and mTld by valine and alanine in both TLLs might be related to change in substrate specificity of these metalloproteases. Except for MMPs the alanine is always present in the second position after the valine. In MMPs that have a broad spectrum of substrates, the two alanines, that are less bulky than valines, do not interfere with the accommodation of various substrates in the cleft of the active center. Thus, the change in the substrate specificity of TLL1 resulting from substitution of the bulkier valine by smaller alanine could allow competition for the binding of nonspecific substrates to the catalytic cleft. This, in turn, could result in lower enzymic efficacy of the mutated TLL1 in the regulation of heart septation during embryonic and fetal development, which would finally lead to the permanent ASD condition.

The CUB3 domain is the only domain present in all $B M P-1$ gene products as well as in both TLLs. A comparison of the sequence flanking the mutated residue in TLL1 shows almost complete conservation of the motif GX(I/L)T(S/T)PGWPKEYPNK (Figure 2c). This signature is also highly conserved among CUB2, 3, and 4 domains in mTld and TLLs with Ile preserved in all the CUBs except in CUB4 of TLL2. Such conservation indicates for functional importance of the peptide in the protein structure. The significance of the change in CUB3 of Val for Ile needs to be verified by obtaining such mutated enzyme and assaying its substrate specificity. Earlier reports indicated that intact structure consisting of CUB3 with both flanking EGF-like motifs are required in mTld for maintaining its activity on specific substrate. ${ }^{39,40}$

The analysis of the TLL1 is, at present, time and labor consuming. The gene consists of $231784 \mathrm{bp}$ divided into 22 exons. The cDNA is $6654 \mathrm{bp}$ long but the ORF consists only $3042 \mathrm{bp}$ and it encodes a polypeptide of 1013 amino acids long. The $5^{\prime}$ upstream sequence of $647 \mathrm{bp}$ is a large $5^{\prime}$ UTR with four methionine codons of an unclear function. The STOP codon is followed by a $230 \mathrm{bp}$ sequence reach in $\mathrm{A}$ and $\mathrm{T}$ stretches. Most of the coding sequence of this gene is enclosed in two large exons, E1 (816bp) and E21/22 (3100 bp). The remaining sequence of $2738 \mathrm{bp}$ is divided into 19 short exons of similar length. There is also a large intron 1, covering more than half of the gene sequence $(116123 \mathrm{bp})$. Its role has yet to be clarified.

Our observations also require, further epidemiological studies on larger and clinically more uniform group of ASD patients to determine the population frequency of mutations in TLL1 gene. The reason that we did not detect defects in all our ASD patients could be explained by the fact that some patients might have mutations in different genes known to be involved in heart development. In addition, familial cases of ASD with TLL1 mutations are yet to be detected in larger population studies that already have begun.

Finally, we have encountered serious problems in obtaining good quality DNA sequence data from the first half of exon 1 and from the large $3^{\prime}$ region of exon 21/22. Thus, it is still possible that in some patients there could be mutations resulting in frame shift and/or nonsense codon mutations. In addition, the promoter and other regulatory regions that we did not sequence in this study could contain mutations affecting gene expression levels. However, here, we have shown for the first time that mutations in TLL1 are linked to the same human syndrome, which was originally generated in transgenic animals by knocking out the gene. ${ }^{13}$ Thus, our results indicate that in some patients with ASD, the genetic causes of the syndrome are heterozygous mutations in TLL1.

\section{Acknowledgements}

The study was supported in part by the Grant no. 2P05D 05927 from the Ministry of Sciences and Education, and by institutional funds NN-1-006/03, NN-4-042/04 and NN-1-014/06.

\section{References}

1 Kahler RL, Braunwald E, Plauth Jr WH, Morrow AG: Familial congenital heart disease: familial occurrence of atrial septal defect with A-V conduction abnormalities, supravalvular aortic and pulmonic stenosis, and ventricular septal defect. Am J Med 1966; 40: $384-399$.

2 Boughman JA, Berg KA, Astemborski JA et al: Familial risks of congenital heart defect assessed in a population based epidemiologic study. Am J Med Genet 1987; 26: 839-849.

3 Van Mierop LHS: Embryology of the atrioventricular canal region and pathogenesis of endocardial cushion defects; in Feldt RH, McGoon DC, Ongley PA, Rastelli GC, Titus JL, Van Mierop LHS (eds): Atrioventricular Canal Defects. Philadelphia, PA: WB Saunders, 1976, pp 1-12.

4 Hoffman JI: Congenital heart disease: incidence and inheritance. Pediatr Clin North Am 1990; 37: 25-43. 
5 Fyler DC: Atrial septal defect secundum; in Fyler DC (ed): Nadas Pediatric Cardiology. Boston, MA: Mosby Year Book Inc., 1992, pp 513-524.

6 Brand A, Keren A, Branski D, Abrahamov A, Stern S: Natural course of atrial septal aneurysm in children and the potential for spontaneous closure of associated septal defect. Am J Cardiol 1989; 64: 996-1001.

7 Zetterqvist P, Turesson I, Johansson BW, Laurell S, Ohlsson NM: Dominant mode of inheritance in atrial septal defect. Clin Genet 1971; $2: 78-86$

8 Pease WE, Nordenberg A, Ladda RL: Familial atrial septal defect with prolonged atrioventricular conduction. Circulation 1976; 53: $759-762$.

9 Li Volti S, Distefano G, Garozzo R, Romeo MG, Sciacca P, Mollica F: Autosomal dominant atrial septal defect of ostium secundum type. Report of three families. Ann Genet 1991; 34: $14-18$.

10 Benson DW, Basson CT, MacRae CA: New understandings in the genetics of congenital heart disease. Curr Opin Pediatr 1996; 8: $505-511$.

11 Johnson MC, Hing A, Wood MK, Watson MS: Chromosome abnormalities in congenital heart disease. Am J Med Genet 1997; 70: $292-298$.

12 Basson CT, Cowley GS, Solomon SD et al: The clinical and genetic spectrum of the Holt-Oram syndrome (heart-hand syndrome). N Engl J Med 1994; 330: 885-891.

13 Clark TG, Conway SJ, Scott IC et al: The mammalian Tolloid-like 1 gene, Tll, 1 is necessary for normal septation and positioning of the heart. Development 1999; 126: 2631-2642.

14 Takahara K, Brevard R, Hoffman GG, Suzuki N, Greenspan DS: Characterization of a novel gene product (mammalian tolloidlike) with high sequence similarity to mammalian tolloid/bone morphogenetic protein-l. Genomics 1996; 34: 157-165.

15 Dumermuth E, Sterchi EE, Jiang W et al: The astacin family of metalloendopeptidases. J Biol Chem 1991; 26: 21381-21385.

16 Mullins MC: Holy Tolloido: Tolloid cleaves SOG/Chordin to free DPP/BMPs. Trends Genet 1998; 14: 127-129.

17 Scott IC, Blitz IL, Pappano WN et al: Mammalian BMP-1/Tolloidrelated metalloproteinases, including novel family member mammalian Tolloid-Like 2, have differential enzymatic activities and distributions of expression relevant to patterning and skeletogenesis. Dev Biol 1999; 213: 283-300.

18 Ge G, Fernández CA, Moses MA, Greenspan DS: Bone morphogenetic protein 1 processes prolactin to a $17-\mathrm{kDa}$ antiangiogenic factor. Proc Natl Acad Sci USA 2007; 104: 10010-10015.

19 Wozney JM, Rosen V, Celeste AJ et al: Novel regulators of bone formation: molecular clones and activities. Science 1988; 242: $1528-1534$

20 Wang EA, Wozney JM: Identification of transforming growth factor 13 family members present in bone-inductive protein purified from bovine bone. Proc Natl Acad Sci USA 1990; 87: 9843-9847.

21 Suzuki N, Labosky PA, Furuta Y et al: Failure of ventral body wall closure in mouse embryos lacking a procollagen C-proteinase encoded by BMP1, a mammalian gene related to Drosophila tolloid. Development 1996; 122: 3587-3595.

22 Yan L, Pollock GH, Nagase H, Sarras Jr MP: A $25.7 \times 10^{3}$ Mr hydra metalloproteinase (HMP1), a member of the astacin family, localizes to the extracellular matrix of Hydra vulgaris in a headspecific manner and has a developmental function. Development 1995; 121: 1591-1602.

23 Childs SR, O'Connor MB: Two domains of the tolloid protein contribute to its unusual genetic interaction with decapentaplegic. Develop Biol 1994; 162: 209-220.

24 Mott JD, Thomas CL, Rosenbach MT, Takahara K, Greenspan DS, Banda MJ: Post-translational proteolytic processing of procollagen C-terminal proteinase enhancer releases a metalloproteinase inhibitor. J Biol Chem 2000; 275: 1384-1390.
25 Goodman SA, Albano R, Wardle FC, Matthews G, Tannahill D, Dale L: BMP1-related metalloproteinases promote the development of ventral mesoderm in early Xenopus embryos. Develop Biol 1998; 195: 144-157.

26 Pappano WN, Scott IC, Clark TG, Eddy RL, Shows TB, Greenspan DS: Coding sequence and expression patterns of mouse chordin and mapping of the cognate mouse Chrd and human CHRD genes. Genomics 1998; 52: 236-239.

27 Piccolo S, Agius E, Lu B, Goodman S, Dale L, De Robertis EM: Cleavage of chordin by xolloid metalloprotease suggests a role for proteolytic processing in the regulation of Spemann organizer activity. Cell 1997; 91: 407-416.

28 Marquès G, Musacchio $M$, Shimell MJ, Wünnenberg-Stapleton K, Cho KW, O'Connor MB: Production of a DPP activity gradient in the early Drosophila embryo through the opposing actions of the SOG and TLD proteins. Cell 1997; 91: 417-426.

29 Panchenko MV, Stetler-Stevenson WG, Trubetskoy OV, Gacheru SN, Kagan HM: Metalloproteinase activity secreted by fibrogenic cells in the processing of prolysyl oxidase. Potential role of procollagen C-proteinase. J Biol Chem 1996; 271: $7113-7119$

30 Takahara K, Kessler E, Biniaminov L et al: Type I procollagen $\mathrm{COOH}$-terminated proteinase enhancer protein: identification, primary structure, and chromosomal localization of the cognate human gene (PCOLCE). J Biol Chem 1994; 269: 26280-26285.

31 Kessler E, Takahara K, Biniaminov L, Brusel M, Greenspan DS: Bone morphogenetic protein-l: the type 1 procollagen C-proteinase. Science 1996; 271: 360-362.

32 Li SW, Sieron A, Fertala A, Hojima Y, Arnold WV, Prockop DJ: The C-proteinase that processes procollagens to fibrillar collagens is identical to the protein previously identified as bone morphogenic protein-1. Proc Natl Acad Sci USA 1996; 93: 5127-5130.

33 Rossant J: Mouse mutants and cardiac development: new molecular insights into cardiogenesis. Circ Res 1996; 78: 349-353.

34 Olson EN, Srivastava D: Molecular pathways controlling heart development. Science 1996; 272: 671-676.

35 Barch MJ, Lawrence HJ, Arsham MS: Peripheral blood culture; in Barch MJ (ed): The ACT Cytogenetics Laboratory Manual, 2nd edition. New York: Raven Press, 1991, pp 17-30.

36 Henegariu O, Heerema NA, Lowe Wright L, Bray-Ward P, Ward DC, Vance GH: Improvements in cytogenetic slide preparation: controlled chromosome spreading, chemical aging and gradual denaturing. Cytometry 1991; 43: 101-109.

37 Church GM, Gilbert W: Genomic sequencing. Proc Nat Acad Sci USA 1984; 81: 1991-1995.

38 Doevendans PA, van Bilsen M: Transcription factors and the cardiac gene programme. Int J Biochem Cell Biol 1996; 28: 387-403.

39 Hartigan N, Garrigue-Antar L, Kadler KE: Bone morphogenetic protein (BMP-1): Identification of the minimal domain structure for procollagen C-proteinase activity. J Biol Chem 2003; 278: $18045-18049$.

40 Garrigue-Antar L, Francois V, Kalder KE: Deletion of epidermal growth factor-like domains converts mammalian tolloid into a chordinase and effective procollagen C-proteinase. J Biol Chem 2004; 279: 49835 - 49841 .

41 Gomis-Rüth FX, Stöcker W, Huber R, Zwilling R, Bode W: Refined $1.8 \AA$ X-ray crystal strucutre of astacin, a zinc-endopeptidase from the crayfish Astacus astacus L. Structure determinantion, refinement, molecular structure and comparison with thermolysin. J Mol Biol 1993; 229: 945-968.

42 Stöcker W, Ng M, Auld DS: Fluorescent oligopeptide substrates for kinetic characterization of the specificity of Astacus protease. Biochemistry 1990; 29: 10418-10425.

43 Garrigue-Antar L, Barker C, Kadler KE: Identification of amino acid residues in bone morphogenetic protein-1 important for procollagen C-proteinase activity. J Biol Chem 2001; 276: $26237-26242$. 